\title{
Survey of Chickpea Rhizobia Diversity in Portugal Reveals the Predominance of Species Distinct from Mesorhizobium ciceri and Mesorhizobium mediterraneum
}

\author{
Print E-mail Add to Marked List Save to EndNote Web. \\ Serviços $9^{\circ}$. Save to EndNote, RefMan, ProCite more options
}

Author(s): Alexandre A (Alexandre, Ana) ${ }^{2}$, Brigido C (Brigido, Clarisse) ${ }^{2}$, Laranjo M (Laranjo, Marta) ${ }^{2}$, Rodrigues S (Rodrigues, Sergio) ${ }^{2}$, Oliveira S (Oliveira, Solange) ${ }^{1}$

Source: MICROBIAL ECOLOGY Volume: 58 Issue: 4 Pages: 930-941 Published: NOV 2009

Times Cited: 0 References: 52 ECitation Map

Abstract: Several Mesorhizobium species are able to induce effective nodules in chickpea, one of the most important legumes worldwide. Our aims were to examine the biogeography of chickpea rhizobia, to search for a predominant species, and to identify the most efficient microsymbiont, considering Portugal as a case study. One hundred and ten isolates were obtained from continental Portugal and Madeira Island. The 16S ribosomal RNA gene phylogeny revealed that isolates are highly diverse, grouping with most Mesorhizobium type strains, in four main clusters (A-D). Interestingly, only $33 \%$ of the isolates grouped with Mesorhizobium ciceri (cluster B) or Mesorhizobium mediterraneum (cluster D), the formerly described specific chickpea microsymbionts. Most isolates belong to cluster A, showing higher sequence similarity with Mesorhizobium huakuii and Mesorhizobium amorphae. The association found between the province of origin and species cluster of the isolates suggests biogeography patterns: most isolates from the north, center, and south belong to clusters $B, A$, and $D$, respectively. Most of the highly efficient isolates (symbiotic effectiveness > 75\%) belong to cluster B. A correlation was found between species cluster and origin soil $\mathrm{pH}$ of the isolates, suggesting that $\mathrm{pH}$ is a key environmental factor, which influences the species geographic distribution. To our knowledge, this is one of the few surveys on chickpea rhizobia and the first systematic assessment of indigenous rhizobia in Portugal.

Document Type: Article

Language: English

KeyWords Plus: RIBOSOMAL-RNA GENE; ARIETINUM-L; SP-NOV; MOLECULAR

CHARACTERIZATION; NATURAL-POPULATIONS; SEQUENCE-ANALYSIS; SYMBIOTIC GENES; DIFFERENT AREAS; STRAINS; TIANSHANENSE

Reprint Address: Oliveira, S (reprint author), Univ Evora, Dept Biol, Apartado 94, P-7002554 Evora, Portugal

Addresses:

1. Univ Evora, Dept Biol, P-7002554 Evora, Portugal

2. Univ Evora, ICAM, Lab Microbiol Solo, P-7002554 Evora, Portugal

E-mail Addresses: ismo@uevora.pt 